\title{
Moral dilemma(s) in human papillomavirus vaccination - revisiting the role of the herd effect
}

Matti Lehtinen ${ }^{1,2}$, Ville N. Pimenoff ${ }^{2}$

1. FICAN-Mid, Tampere University Hospital, Tampere, Finland

2. Department of Laboratory Medicine, Karolinska Institute, Stockholm, Sweden

Correspondence: Matti Lehtinen (matti.lehtinen@tuni.fi)

Citation style for this article:

Lehtinen Matti, Pimenoff Ville N.. Moral dilemma(s) in human papillomavirus vaccination - revisiting the role of the herd effect. Euro Surveill.

2021;26(50): $\mathrm{pii}=2101154$. https://doi.org/10.2807/1560-7917.ES.2021.26.50.2101154

Article submitted on 15 Dec 2021 / accepted on 16 Dec 2021 / published on 16 Dec 2021

In this Eurosurveillance issue, two articles by Colzani et al. [1] and Ortu et al. [2], raise the question of who should be prioritised in vaccination policy when there is a temporary global shortage of vaccines. These articles on human papillomavirus (HPV) are a reminder that discussions pertaining to vaccination policies are not confined to coronavirus disease (COVID-19). Nonetheless, lessons learnt from the establishment of HPV vaccination are generalisable and valuable for the implementation and monitoring of most vaccination programmes, including those against COVID-19, worldwide.

Colzani et al. [1] use the eradication of rubella as an example of beneficial gender-neutral vaccination. Indeed, together with cost-efficiency of offering HPV vaccination to both girls and boys, already at moderate coverage, the subsequently achieved herd effect (i.e. indirect protection to the unvaccinated in a population) is very important. In the past, eradication attempts with girls-only rubella virus vaccination failed in the Nordic countries. However, rubella - as well as mumps - was eradicated from the Nordic countries in just 15 years (between 1982 and 1997) after the launch of a gender-neutral measles-mumps-rubella (MMR) vaccination programme [3]. Girls-only rubella vaccination with $70 \%$ coverage in the 1970 s only moved the peakage of rubella incidence to fertile-aged women without any effect on the incidence of congenital rubella syndrome. Hereafter, eradication was achieved only once high coverage of gender-neutral MMR vaccination was achieved and followed by a strong herd effect. Measles, which has a high basic reproduction number (Ro) of 10, was also essentially eradicated from the Nordic countries through this programme, and the status is still retained [4].

Among the more than 300 different HPVs infecting humans, types $16,18,31$ and 45 are the most oncogenic viral agents for humans with over $90 \%$ of the
HPV-associated cancer burden. The elimination of HPVassociated cancers will be accomplished by herd effect enforced eradication of these most oncogenic and prevalent HPV types. Because of both, the much lower Ro, less than 4 for all other HPV types than HPV16, and the assortativeness of sexual risk-taking behaviour, it is the herd effect from moderate to high coverage gender-neutral vaccination that is the relevant factor providing protection even against HPV16 [5,6]. A community-randomised study has shown that the herd effect is notable in a real-life setting within less than 4 years but only after gender-neutral vaccination $[5,6]$. In addition, most mathematical models do not account for this, and thus their cost-efficiency estimates for the gender-neutral HPV vaccination could (should) be even better.

Colzani et al. [1] meticulously go through the European HPV vaccination policies. They contemplate whether or not it is morally right to prioritise offering HPV vaccine to girls in low- and middle-income countries (LMICs) with high cervical cancer incidence or if offering vaccination to the entire population of the young is morally right. Ortu et al. [2] report on low HPV vaccination coverage in another risk group, men who have sex with men. In this respect, it is of note that countries will always be left with particular group(s) of unvaccinated individuals. Herd effect, which stems rapidly and efficiently from gender-neutral HPV vaccination, also protects those risk groups with low uptake of the offered HPV vaccine [7]. The herd effect induced by even moderate gender-neutral vaccination coverage is thus crucial to protect the most vulnerable who are not vaccinated. The moral aspect here is that when advocating, accepting, or implementing any prophylactic vaccination policy, one is not only protecting the individual (ourselves) but the community as well (others).

Most importantly, it may not be possible to easily circumvent HPV vaccine shortage by changing the vaccination 
schedule or dosing. No HPV vaccine-induced protective antibody level has been defined and overall, the effect from one-dose vaccinations, aside from that with live vaccines, is unclear and assumes boostering which has its caveats (e.g. likely limited access to and availability of services) in the LMICs. Studies have shown that up to $10 \%$ of the 4 -valent HPV vaccine recipients do not have neutralising or total HPV18 L1-VLP (virus-like particle) antibodies 7 to 12 years post vaccination $[8,9]$, which explains the less than $100 \%$ vaccine efficacy against invasive cervical cancer [10]. Furthermore, high-quality health registry-based data showed that HPV vaccine efficacy against immediate cervical precancer (cervical intraepithelial neoplasm $(\mathrm{CIN}) 3+$ ) from one-dose vaccination is significantly worse than from three-dose vaccination [11]. Taken together, attempts to solve the moral dilemma caused by temporary vaccine shortage should not cause new moral conflicts.

\section{Conflict of interest}

ML has previously received grants from Merck \& Co. Inc. and the GSK group of companies through his then employer (The University of Tampere).

\section{References}

1. Colzani E, Johansen K, Johnson H, Pastore Celentano L. Human papillomavirus vaccination in the European Union/European Economic Area and globally: a moral dilemma. Euro Surveill. 2021;26(50):2001659. https://doi.org/10.2807/1560-7917. ES.2021.26.50.2001659

2. Ortu G, Barret A-S, Danis K, Duchesne L, Levy-Bruhl D, Velter A. Low vaccination coverage for human papillomavirus disease among young men who have sex with men, France, 2019. Euro Surveill. 2021;26(50):2001965. https://doi.org/10.2807/15607917.ES.2021.26.50.2001965

3. Peltola H, Davidkin I, Paunio M, Valle M, Leinikki P, Heinonen OP. Mumps and rubella eliminated from Finland. JAMA. 2000;284(20):2643-7. https://doi.org/10.1001/ jama.284.20.2643 PMID: 11086376

4. World Health Organization Regional Office for Europe (WHO/ Europe). Measles and rubella elimination: verification process continues amid COVID-19 pandemic. Copenhagen: WHO/ Europe; 13 Aug 2020. Available from: https://www.euro.who. int/en/countries/sweden/news/news/2020/8/measles-andrubella-elimination-verification-process-continues-amid-covid19-pandemic

5. Vänskä S, Luostarinen T, Baussano I, Apter D, Erikson $\mathrm{T}$, Natunen K, et al. Vaccination with moderate coverage eradicates oncogenic HPV if a gender-neutral strategy is applied. J Infect Dis. 2020;222:948-56. https://doi. org/10.1093/infdis/jiaa099 PMID: 32161969

6. Gray P, Kann H, Pimenoff VN, Eriksson T, Luostarinen T, Vänskä $S$, et al. HPV seroprevalence in pregnant women following gender-neutral and girls-only vaccination programs in Finland: A Cross-sectional cohort analysis following a clusterrandomised trial. PLoS Med. 2021;18:e1003588. https://doi. org/10.1371/journal.pmed.1003588 PMID: 34097688

7. Malmqvist E, Helgesson G, Lehtinen J, Natunen K, Lehtinen M. The ethics of implementing HPV vaccination. Med Health Care Philos. 2011;14:19-27. https://doi.org/10.1007/s11019-0109285-9 PMID: 20853152

8. Mariz FC, Gray P, Bender E, Eriksson T, Kann H, Apter D, et al. Sustainability of bi- and quadrivalent HPV vaccine-induced neutralizing antibodies. Lancet Infect Dis. 2021;10:1458 68. https://doi.org/10.1016/S1473-3099(20)30873-2 PMID: 34081923

9. Kann H, Lehtinen M, Eriksson T, Surcel H-M, Dillner J, Faust $H$. Sustained cross-reactive antibody responses after human papillomavirus vaccinations. Up to 12 years follow-up in the Finnish Maternity Cohort. J Infect Dis. 2021;223(11):1992-2000. https://doi.org/10.1093/infdis/jiaa617 PMID: 33009576

10. Lei J, Ploner A, Elfström KM, Wang J, Roth A, Fang F, et al. HPV vaccination and the risk of invasive cervical cancer. $N$
Engl J Med. 2020;383(14):1340-8. https://doi.org/10.1056/ NEJMoa1917338 PMID: 32997908

11. Gargano JW, You M, Potter R, Alverson G, Swanson R, Saraiya M, et al. An evaluation of dose-related HPV vaccine effectiveness using central registries in Michigan. Cancer Epidemiol Biomarkers Prev. 2021; cebp.0625.2021. PMID: 34663615

\section{License, supplementary material and copyright}

This is an open-access article distributed under the terms of the Creative Commons Attribution (CC BY 4.0) Licence. You may share and adapt the material, but must give appropriate credit to the source, provide a link to the licence and indicate if changes were made.

Any supplementary material referenced in the article can be found in the online version.

This article is copyright of the authors or their affiliated institutions, 2021. 